\title{
Towards a New Classification of Location Privacy Methods in Pervasive Computing
}

\author{
Mads Schaarup Andersen and Mikkel Baun Kjærgaard \\ Department of Computer Science \\ Aarhus University \\ \{masa, mikkelbk\}@cs.au.dk
}

\begin{abstract}
Over the last decade many methods for location privacy have been proposed, but the mapping between classes of location based services and location privacy methods is not obvious. This entails confusion for developers, lack of usage of privacy methods, and an unclear roadmap ahead for research within location privacy. This paper presents a two-dimensional classification of existing methods for location privacy grouping them by the type of location based service to which they apply and location privacy method category. The types of location based services identified are Point-of-Interest, Social Networking, Collaborative Sensing, and Route Tracing, and the high level location privacy method categories are Anonymization, Classical Security, Spatial Obfuscation, Temporal Obfuscation, and Protocol. It is found that little work exists on location privacy in the areas of Social Networking and Collaborative Sensing, and that insufficient work has been done in Route Tracing. It is concluded that none of the existing methods cover all applications of Route Tracing. It is, therefore, suggested that a new overall method should be proposed to solve the problem of location privacy in Route Tracing. Furthermore, future challenges are identified.
\end{abstract}

Key words: Location Privacy, Pervasive Computing, Ubiquitous Computing

\section{Introduction}

With the number of smart phones based on Android and iOS soon exceeding the number of stationary and laptop computers on the Internet, applications for these devices, usually referred to as Apps, are penetrating the everyday life of people. One of the interesting aspects of Apps is that they are able to incorporate sensor data from the device and use it to provide services based on this. Currently, many apps are available for these devices and a subset of these use the location of the device (computed in some way using one or more sensors) as an integrated and essential part. These are called location based services (LBSs). In February 2010, there were 6,400 LBSs in Apples AppStore and more than 1,000 LBSs in Androids Marketplace ${ }^{1}$. Using location as an integrated part of

\footnotetext{
${ }^{1}$ http://skyhookwireless.com/locationapps/
} 
an application has enabled a number of new application domains such as navigation, friend finder, route tracking, etc. However, these services require the user to disclose his location. This introduces the concern of preserving the privacy of the user. In 1993, Bellotti and Sellen recognized that ubiquitous computing is particularly prone to attack on privacy [5] and in 2001, Langheinrich proposed six guidelines to system design to include privacy concerns [26]. Since then a lot of different methods for location privacy have been introduced, but each of them for a specific application domain and usually to solve a very specific problem. This makes it difficult for a developer to choose how location privacy should be obtained, and hence location privacy methods are often not added. Furthermore, there seems to be a lack of work in location privacy for other than Point-of-Interest (POI) services, e.g., GPS navigation, where a user can query a service for route to an address. However, during recent years new types of LBSs have emerged where the usage of location has become increasingly complex. Position is no longer shared only with a service, but might also be shared with other users in real time. Furthermore, other things than just the position might be shared with the position receiver making things such as recommender services possible. This makes it interesting to categorize LBSs in relation to location privacy, and thereafter place the existing location privacy methods using this categorization.

The contribution of this paper is twofold. The first contribution is to provide an overview of how existing location privacy methods relate to different types of LBSs, hence aiding the developer in the choice of privacy method. This is done using a two-dimensional classification scheme with type of $L B S$ on one axis and high level location privacy method on the other. The existing work is then placed in this categorization, and hence a developer can consult it to choose an appropriate method for his location enabled app. The second contribution of this paper is to provide the research community with a road-map for research in location privacy. This is done by analyzing the above mentioned overview to reveal gaps in research. It will then be considered whether the location privacy methods of the classification can solve the gaps, and if this it is the case that a category has issues that cannot be solved with existing methods, a new method will be proposed.

Several other papers have addressed the problem of surveying and classifying location privacy methods. Krumm [25] identifies a number of open research questions in location privacy by identifying location privacy methods, attacks on these, and by surveying the results of empirical studies. Scipioni and Langheinrich [34] recognize the need for further research to be done in relation to location privacy in the newer LBS category of Social Networking (SN) services, and they propose a categorization of LBSs based on the multiplicity between sender and receiver of location. Christin et. al [8] survey the entire area of privacy in participatory sensing including location privacy. However, the three mentioned papers lack a way to make the coupling between existing methods and how they can be applied to different categories of LBSs. 
The rest of the paper is structured as follows. Section 2 and Section 3 describe the two dimensions of the classification. Section 4 places the existing methods in the classification. Section 5 covers open challenges and Section 6 provides a conclusion.

\section{Categories of Methods for Location Privacy}

In the following we present our categorization of location privacy methods. This is based one a categorization proposed by Andersen [3], which builds on the work of Duckham and Kulik [12]. In this paper we divide privacy methods into the five categories of: Anonymity, Classical Security, Spatial Obfuscation, Temporal Obfuscation, and Protocol. We elaborate on these categories in the following:

Anonymity methods obtain privacy by hiding the identity of the user when providing the location. It is therefore possible to see the exact location, but impossible to tie any user to the location. A sub-category of anonymity is pseudonymity where the user's real identity is replaced by a pseudonym, and the idea is then that it should be impossible for an adversary to use this pseudonym to identify the user's real identity.

Classical Security methods provide privacy using classical security methods. This can be either cryptography or security policies. In cryptography the position can be encrypted using either a secret or public key encryption scheme (usually public key), so that only the provider and consumer of the position will be able to read the contents, hiding it from adversaries. When using security policies it can be expressed who can and cannot gain access, similar to access control lists in file systems.

Spatial Obfuscation methods obtain privacy by obfuscation the position of the user in space. This means that rather than providing a single point as the location an area $A$ is provided to the position receiver.

Temporal Obfuscation methods provide privacy by obfuscating the position of the user in time. This means that instead of providing the user position in real time, the location is provided at an obfuscated position in time by adding $\Delta t$.

Protocol Privacy is obtained by having a custom protocol to enhance the privacy. This is usually used in combination with anonymity or spatial obfuscation to either hide the user identity or aid in obfuscating the position.

\section{Categories of Location Based Services}

In this section we will provide a categorization of LBSs. The categorization is defined in relation to relevant properties of location privacy methods. This is 
partly inspired by Andersen [3] who proposes LBS categories and Scipioni and Langheinrich [34] who divide SN services into categories based on multiplicity between sender and receiver. It is our claim that the latter applies to a broader range of LBSs than just SN. Besides that we include details about the position sensitivity, importance of ID, and whether the position is shared in real time. We use this to emphasize what differs between the types of LBS that we identified. The interesting properties are:

Receiver Who is receiving the position? This can either be a service which is running on a server or it can be peers where other users will use the position. A last option would be the case where the service runs locally, as it is the case in most car navigation devices. However, if everything runs locally the privacy concerns change. If the phone is stolen it is suddenly a threat to the privacy if sensitive information is located on the phone. This is, however, not in the scope of the privacy of intended sharing of positions and is therefore omitted.

Number of Receivers The number of users which will receive the position. Can be 1 or $n$.

Number of Users The number of users which need to provide their position in order for the LBS to make sense. Can be 1 or $n$.

ID Importance How important is it for the LBS to know the true identity of the user. Can be High, Medium, or Low. High means very important, medium means that it depends on the specific application, and low means that it is not needed.

Spatial Sensitivity How important is it to place the user in an exact point rather than an area. Can be High, Medium, or Low. High means that exact position is needed, medium means that it depends on the specific application, and low means that an area can be provided instead.

Temporal Sensitivity How important is it that the user is placed in time. Can be High, Medium, or Low. High means that the LBS needs the time, medium means that it depends on the specific application, and low means that time is never essential.

Sharing Phase Is the data shared in real time, or at a later stage. Referred to as online (real time) and offline phases.

In our categorization of LBSs, we will call the simple request-reply applications Point-of-Interest (POI), everything concerned with social networks Social Networking (SN), and participatory sensing Collaborative Sensing (CS) as we in the CS category focus on the type of LBSs where users share data with everybody. Furthermore, none of the three mentioned categories cover an emerging type of LBS, namely Route Tracing (RT). In this category of LBSs, instead of sharing a single position, the user shares a trace of coherent positions. The four categories are explained in detail in the following. Table 1 lists the important properties in relation to location privacy for the four mentioned categories. 


\begin{tabular}{|l|l|l|l|l|}
\hline & POI & SN & CS & RT \\
\hline Receiver & Service & Peers & Service & Peers or Service \\
\hline \# Receivers & 1 & $n$ & $n$ & $n$ \\
\hline \# Users & 1 & 1 & $n$ & 1 \\
\hline ID Importance & High & High & Low & Medium \\
\hline Spatial Sen. & High & Medium & High & Medium \\
\hline Temporal Sen. & High & High & High & Medium \\
\hline Sharing Ph. & Online & Online & Online or Offline & Offline \\
\hline
\end{tabular}

Table 1. Important location privacy properties of the four categories of LBSs.

Point-of-Interest POI services are the first LBSs which appeared. Here a user sends a request to a server for nearest POI. This can e.g. be gas stations or the local weather forecast, and the service then replies with a set of coordinates, localized information, or possibly a route direction to the POI. In POI services, a single user queries a server to find the nearest POI. I.e. we have a 1 to 1 relationship between users and receivers. As the server needs to know who to send the reply to, ID has high importance. POI services are highly spatial sensitive as the precision of the reply depends on it. When the user is moving, POI services are, furthermore, very sensitive in regards to temporal aspects. POI services are only relevant in an online phase.

Social Network Services SN services have become popular over the last few years with the wide adaption of Facebook Places ${ }^{2}$, Google Latitude ${ }^{3}$ etc. In these services, users share locations (and optionally meta-data) with friends, which are considered peers. SN services have a 1 to $n$ relationship between providers and consumers, as the provider wants to share his data with a well defined (sharing with all is also considered well defined) set of receivers. As SN services are in nature self-promoting, the ID of the user is very important. Spatially, the sensitivity is of medium importance, as it is only important to tie the user to a place which might be defined as an overall area, e.g., in Facebook places where the user's location might be a building complex. Temporal sensitivity is also high as it is not interesting for receivers to know that the user at some point was at a concert if the receivers are trying to meet up with the person. SN services are interesting in an online phase.

Collaborative Sensing In CS services, the users collect data and provide them for a service and potentially all other users. An example of this is a traffic monitoring system such as the one provided by Google Maps ${ }^{4}$. CS services have an $n$ to $n$ relationship between sender and receivers as it is vital that all the users participate for the service to make sense, and that everybody can access the data. The ID of the individual user is of low importance as it is rather the

\footnotetext{
${ }^{2}$ http://www.facebook.com/places

${ }^{3}$ http://www.google.com/latitude

${ }^{4}$ http://maps.google.com
} 
data he provides that is relevant to the service. Spatially and temporally the sensitivity is high as the exact position is needed as well as the position in time is important. CS services are interesting in both online and offline cases. This is illustrated by the traffic monitoring example. Here the data can be used to get a real time image of the traffic at a certain point, but the data can also be used for analysis of areas often prone to congestion.

Route Tracing The above categories cover all LBSs where a position is shared in real-time, or in an online phase. However, if we want to share a trace of positions collected earlier, in an offline phase, we have another set of privacy challenges. In RT the users collect a trace of positions linked in time rather than a single position. An example is sharing exercises such as running routes in systems such as Endomondo ${ }^{5}$ where users then can compete on the same routes and distance and analyze performance. Furthermore, RT is becoming widely adapted by insurance companies offering usage-based insurance. An example of this is Alkabox ${ }^{6}$ where a box records the GPS position in conjunction with acceleration data and calculates insurance based on these in case of an accident. $\mathrm{RT}$ is a relatively new type of LBS but is very important as not only are people sharing exercise routes, but companies are starting to record data about their customers. Furthermore, it has been proposed to use RT for road pricing [11] and hence the government might be able to track citizens leading to a big brother scenario. It is therefore the opinion of the authors that the area of RT is of high importance. That RT should be a category on its own has not yet been proposed in the literature, but as it has different properties than the rest of the categories proposed, it is indicated that RT is not covered by any of the other categories.

In $\mathrm{RT}$, the trace is shared with peers or a service, and the multiplicity between senders and receivers is 1 to $n$. ID in itself has medium importance depending on the specific application domain. In insurance it is necessary for the insurer to know the ID, but in exercise sharing it is less important to know the exact ID of the user. Here, characteristics of the user might be enough. Spatially, the precision needed is medium as the appropriate accuracy of the position depends on the application area. Temporally, the sensitivity is also medium as it is important to be able to place the individual positions in relation to each other. However, the positions might not need to be placed in time of day. RT is done in an offline phase.

\section{Classification of Existing Methods}

In the following section, we will classify the existing location privacy methods with respect to the two above mentioned dimensions. Thereafter, we will analyze the contents of the table focusing on empty or close to empty cells and reason about whether the gap has a logical explanation or it is an open area

\footnotetext{
${ }^{5}$ http://www.endomondo.com

${ }^{6}$ http://www.alkabox.dk
} 
needing more research. The result of the classification can be found in Table 2 . A challenge for the classification is that some methods combine several types of privacy methods. An example is CacheCloak [29] which is a combination of anonymity and temporal obfuscation. The papers where this is the case, are placed in the category where they fit the best by the authors judgment. In the case of CacheCloak this is anonymity. To discuss the result of the classification

\begin{tabular}{|c|c|c|c|c|}
\hline & Point-of-interest & \begin{tabular}{|ll}
$\begin{array}{l}\text { Social } \\
\text { working }\end{array}$ & Net- \\
\end{tabular} & $\begin{array}{l}\text { Collaborative } \\
\text { Sensing }\end{array}$ & Route Tracing \\
\hline Anonymity & $\begin{array}{l}\text { Mix Zones[6] } \\
\text { k-anonymity[15] } \\
\text { CacheCloak[29] } \\
\text { CliqueCloak[14] } \\
\text { Distributed } \\
\text { Anonymity[18] } \\
\text { Pseudonymity[33] }\end{array}$ & & $\begin{array}{l}\text { AnonySense[9] } \\
\text { Traffic } \\
\text { Monitoring[19] } \\
\text { HitchHiking[35] }\end{array}$ & \\
\hline Security & $\begin{array}{l}\text { P3P Inspired[1] } \\
\text { Confab[22] } \\
\text { Dynamic Privacy } \\
\text { Management[21] } \\
\text { LocServ[32] }\end{array}$ & Loccacino[10] & & \\
\hline Spatial Ob. & $\begin{array}{l}\text { LBAC[4] } \\
\text { SpaceTwist[36] } \\
\text { Grid-based[16] } \\
\text { KNN[23] } \\
\text { Source } \\
\text { Simulation[28] } \\
\text { Louis, Lester and } \\
\text { Pierre[38] } \\
\text { ILRQ[7] } \\
\text { New Casper[30] } \\
\text { Obfuscation[13] } \\
\text { Path } \\
\text { Confusion[20] }\end{array}$ & $\begin{array}{l}\text { Proximity } \\
\text { Queries[27] }\end{array}$ & & \begin{tabular}{|lr} 
Gaussian & Noise \\
{$[24]$} & \\
Spatial & Rounding \\
{$[24]$} & \\
Selective & Hiding \\
{$[31]$} &
\end{tabular} \\
\hline Temporal Ob. & Temporal Ob.[17] & & & \\
\hline Protocol & $\begin{array}{l}\text { MIST[2] } \\
\text { Geographic } \\
\text { Routing[37] }\end{array}$ & & & \\
\hline
\end{tabular}

Table 2. Classification of existing location privacy methods to high level location privacy methods and categories of LBSs.

we start out with the filled cells:

A lot of work has been done on anonymization in POI [6, 15, 29, 14, 18, 33]. An example is k-anonymity [15] where the user is indistinguishable among $k-1$ other users. Classical security in POI is also well covered [1, 22, 21, 32]. An example is Confab [22] which is a framework providing basic security mechanisms 
for controlling access to data. Spatial obfuscation has also been a main area of interest in POI [4, 36, 16, 23, 28, 38, 7, 30, 13, 20, 27]. The New Casper [30] is an example where the user is placed in a grid structure rather than in a single point. Though, not a lot of work has been done in temporal obfuscation, Gruteser and Grunwald [17] have made comments on how such a method should work. Finally, in POI work has been done in creating custom privacy enabling protocols $[2,37]$. An example is MIST [2] where the routing of the data makes it very difficult to track the origin of data.

In classical security for $\mathrm{SN}$, some work has been done in the Loccacino project [10]. Here user controllable privacy has been tested, mainly with the use of access control lists. Within SN there has also been work in spatial obfuscation with proximity queries [27] where a threshold is used to obfuscate the position of the user.

Some work has been done in anonymization for CS $[9,35,19]$. An example of this is Hitchhiking [35] where the user's ID is hidden.

Lastly, there has been some work done on spatial obfuscation for RT [24, 31]. An example of this is adding Gaussian Noise to a trace, hence rendering it impossible to distinguish the actual positions from the added noise [24].

Next, we will comment on the obvious empty cells in the table. One of the first things to notice is that close to no work has been done in temporal obfuscation for any category. With Table 1 in mind, this is explainable with the fact that all LBSs have high or medium temporal sensitivity, and hence the method is not well suited for LBSs. The next thing to notice in the table is that the POI category is well covered in all location method categories, and hence it should be easy for a developer to choose an appropriate method for any kind of application within this LBS category. Moreover, it seems that this area is well researched, as a large variety of methods have been proposed.

In relation to SN services, not that many methods have been proposed. Here the anonymization category is empty. In relation to our definition of SN services, this makes sense, as the purpose is for the user to share some information about himself. If the data was anonymized it would be impossible to tie this information to a user. The fact that there has only been done work in the categories of security and spatial obfuscation indicates that these categories fit well to SN, but protocol seems to be an unexplored area with possibilities.

In the CS category, there has only been published anonymization location privacy methods. This is due to the fact that when data is anonymized it is difficult to infer a specific user, and since these applications cover LBSs where user ID is irrelevant, this make sense. Sometimes it might, however, make sense to apply some of the other methods to further enhance the privacy of the participating user. This could e.g. be in a weather sensing application where an area rather than a point might be enough. Hence, CS also has unexplored areas.

Lastly, we see that RT only has work done in spatial obfuscation [24, 31]. This works fine for some applications of RT, but for the applications mentioned earlier in Section 3 these methods have limited applicability. This is e.g. the case for 
usage based insurance. Snapping to a grid, as done in Spatial Rounding, might hide the fact that the user has driven in a certain high-risk area which would increase the insurance (depending on the insurance policy and resolution of the grid). In adding Gaussian noise there might be the problem that the insurance company would charge the user for being in all positions (including the noise), and hence this has a potentially huge disadvantage for the user. Lastly, using selective hiding the user could choose to always hide a specific high-risk area in his trace.

As mentioned earlier, RT is becoming more and more used, and considering the mentioned possible applications it is very important to develop privacy methods which cover these as well. An observation is that other categories of LBSs are concerned with sharing a single position and RT is concerned with sharing an entire trace of positions. This observation suggests that it might be a good idea to do some research in an entirely new high level method for location privacy.

\section{Open Challenges}

As identified in this paper there are still a lot of open challenges in the area of location privacy. We see challenges in three main areas: Location Privacy Methods, Software Engineering, and User Awareness.

Location Privacy Methods require more exploration of protocol and temporal obfuscation for SN services; spatial and temporal obfuscation, protocol, and classical security for CS; and all areas of RT. Moreover, we suggest that emphasis is put on developing a new category for RT as RT has many features that differ from the other categories of LBSs. This is mainly due to the fact that a trace rather than a single position is shared. It is a challenge that for some domains of RT spatial rounding, adding noise, or using selective hiding are not applicable.

Software Engineering calls for further examination of how application developers can be aided in adding location privacy to applications. The categorization presented in this paper is the first step, but currently no tools or frameworks that can aid the developer in adding location privacy to LBSs exist. The ones that do exist are only concerned with very specific types of LBSs in mind. It would be interesting to examine the effects of providing application developers with such a tool or framework, and see if it has an impact of how often location privacy methods would be used.

User Awareness is problematic as users are not all that concerned about sharing their location with others. This is the case, even though research has shown the potential dangers of doing so. Futhermore, research needs to be done in how we make people aware of the possible dangers of sharing location data. It seems that a demand from users could drive the developers to care more. 


\section{Conclusion}

In this paper we presented a two-dimensional classification of location privacy methods in relation to types of LBSs. In this classification we placed the existing privacy methods proposed by the research community. This will serve as a reference for LBS developers wanting to add privacy support to applications. Furthermore, the classification had the purpose of pointing out open areas in location privacy research. The results were that POI is fully covered and less research should be focused on this area. However, there are open issues in the areas of SN and CS services where more privacy methods should be explored. Moreover, the classification showed that the area of RT, though important, is close to unexplored, and that the methods proposed do not cover all applications of RT, suggesting that none of the high level location privacy methods fits the specific purpose. It is concluded that a new category of location privacy methods is needed for this RT.

\section{References}

1. Mark S. Ackerman. Privacy in pervasive environments: next generation labeling protocols. Personal Ubiquitous Comput., 8:430-439, November 2004.

2. J. Al-Muhtadi, R. Campbell, A. Kapadia, M.D. Mickunas, and Seung Yi. Routing through the mist: privacy preserving communication in ubiquitous computing environments. In Distributed Computing Systems, 2002. Proc. 22nd Int. Conference on, pages $74-83,2002$.

3. M. S. Andersen. On limitations of existing methods for location privacy. 3rd International Workshop on Security and Privacy in Spontaneous Interaction and Mobile Phone Use, 2011.

4. C. A. Ardagna, M. Cremonini, E. Damiani, S. De Capitani di Vimercati, and P. Samarati. Location privacy protection through obfuscation-based techniques. In Proc. of the 21st annual IFIP WG 11.3 working conf. on Data and applications security, pages 47-60, Berlin, Heidelberg, 2007. Springer-Verlag.

5. Victoria Bellotti and Abigail Sellen. Design for privacy in ubiquitous computing environments. In Proc. of the 3rd conf. on European Conference on ComputerSupported Cooperative Work, pages 77-92, Norwell, MA, USA, 1993. Kluwer Academic Publishers.

6. A.R. Beresford and F. Stajano. Location privacy in pervasive computing. Pervasive Computing, IEEE, 2(1):46 - 55, 2003.

7. Reynold Cheng, Yu Zhang, Elisa Bertino, and Sunil Prabhakar. Preserving user location privacy in mobile data management infrastructures. 2006.

8. Delphine Christin, Andreas Reinhardt, Salil Kanhere, and Matthias Hollick. A survey on privacy in mobile participatory sensing applications. Journal of Systems and Software, In Press, Accepted Manuscript, 2011.

9. Cory Cornelius, Apu Kapadia, David Kotz, Dan Peebles, Minho Shin, and Nikos Triandopoulos. Anonysense: privacy-aware people-centric sensing. In Proc. of the 6th Int. Conf. on Mobile systems, applications, and services, MobiSys '08, pages 211-224, New York, NY, USA, 2008. ACM. 
10. Jason Cornwell, Ian Fette, Gary Hsieh, Madhu Prabaker, Jinghai Rao, Karen Tang, Kami Vaniea, Lujo Bauer, Lorrie Cranor, Jason Hong, Bruce McLaren, Mike Reiter, and Norman Sadeh. User-controllable security and privacy for pervasive computing. In Proc. of the 8th IEEE Workshop on Mobile Computing Systems and Applications, 2007.

11. Vlad Coroama. The smart tachograph - individual accounting of traffic costs and its implications. In Kenneth Fishkin, Bernt Schiele, Paddy Nixon, and Aaron Quigley, editors, Pervasive Computing, volume 3968 of Lecture Notes in Computer Science, pages 135-152. Springer Berlin / Heidelberg, 2006.

12. M. Duckham and L. Kulik. Drummond J (ed) Dynamic $\&$ mobile GIS: investigating change in space and time. CRC, 2006.

13. Matt Duckham and Lars Kulik. A formal model of obfuscation and negotiation for location privacy. In Hans W. Gellersen, Roy Want, and Albrecht Schmidt, editors, Pervasive Computing, volume 3468 of Lecture Notes in Computer Science, pages 152-170. Springer Berlin / Heidelberg, 2005.

14. B. Gedik and Ling Liu. Location privacy in mobile systems: A personalized anonymization model. In Proc. of the 25th IEEE Int. Conf on Distributed Computing Systems, pages $620-629,2005$.

15. Bugra Gedik and Ling Liu. A customizable k-anonymity model for protecting location privacy. In In ICDCS, pages 620-629, 2004.

16. G. Gidofalvi, Xuegang Huang, and T.B. Pedersen. Privacy-preserving data mining on moving object trajectories. In Mobile Data Management, 2007 Int. Conf. on.

17. Marco Gruteser and Dirk Grunwald. Anonymous usage of location-based services through spatial and temporal cloaking. In Proceedings of the 1st international conference on Mobile systems, applications and services, MobiSys '03, pages 3142, New York, NY, USA, 2003. ACM.

18. Marco Gruteser, Graham Schelle, Ashish Jain, Rick Han, and Dirk Grunwald. Privacy-aware location sensor networks. In Proc. of the 9th conf. on Hot Topics in Operating Systems - Volume 9, 2003.

19. B. Hoh, M. Gruteser, H. Xiong, and A. Alrabady. Enhancing security and privacy in traffic-monitoring systems. Pervasive Computing, IEEE, 5(4):38 -46, 2006.

20. Baik Hoh and M. Gruteser. Protecting location privacy through path confusion. In Security and Privacy for Emerging Areas in Communications Networks, 1st Int. Conf. on, 2005.

21. Dan Hong, Mingxuan Yuan, and Vincent Y. Shen. Dynamic privacy management: a plug-in service for the middleware in pervasive computing. In Proc. of the 7th int. conf. on Human computer interaction with mobile devices $\mathscr{G}$ services, 2005.

22. Jason I. Hong and James A. Landay. An architecture for privacy-sensitive ubiquitous computing. In Proceedings of the 2nd international conference on Mobile systems, applications, and services, MobiSys '04, pages 177-189, New York, NY, USA, 2004. ACM.

23. Ali Khoshgozaran and Cyrus Shahabi. Blind evaluation of nearest neighbor queries using space transformation to preserve location privacy. In Proc. of the 10th int. conf. on Advances in spatial and temporal databases, 2007.

24. John Krumm. Inference attacks on location tracks. In Proc. of the 5th int. conf. on Pervasive computing, 2007.

25. John Krumm. A survey of computational location privacy. Personal Ubiquitous Comput., 13:391-399, August 2009.

26. Marc Langheinrich. Privacy by design - principles of privacy-aware ubiquitous systems. In Proc. of the 3rd int. conf. on Ubiquitous Computing, 2001. 
27. Sergio Mascetti, Claudio Bettini, Dario Freni, X. Sean Wang, and Sushil Jajodia. Privacy-aware proximity based services. In Proc. of the 10th Int. Conf. on Mobile Data Management: Systems, Services and Middleware, 2009.

28. K. Mehta, Donggang Liu, and M. Wright. Location privacy in sensor networks against a global eavesdropper. In Network Protocols, IEEE Int. Conf. on, 2007.

29. Joseph Meyerowitz and Romit Roy Choudhury. Hiding stars with fireworks: location privacy through camouflage. In Proc. of the 15th annual int. conf. on Mobile computing and networking, 2009.

30. Mohamed F. Mokbel, Chi-Yin Chow, and Walid G. Aref. The new casper: query processing for location services without compromising privacy. In Proc. of the 32nd int. conf. on Very large data bases, 2006.

31. Min Mun, Sasank Reddy, Katie Shilton, Nathan Yau, Jeff Burke, Deborah Estrin, Mark Hansen, Eric Howard, Ruth West, and Péter Boda. Peir, the personal environmental impact report, as a platform for participatory sensing systems research. In Proc. of the rth int. conf. on Mobile systems, applications, and services.

32. Ginger Myles, Adrian Friday, and Nigel Davies. Preserving privacy in environments with location-based applications. IEEE Pervasive Computing, 2:56-64, January 2003.

33. Andreas Pfitzmann and Marit Köhntopp. Anonymity, unobservability, and pseudeonymity; a proposal for terminology. In Int. workshop on Designing privacy enhancing technologies: design issues in anonymity and unobservability, 2001.

34. Marcello Paolo Scipioni and Marc Langheinrich. I'm here! privacy challenges in mobile location sharing. 2nd Int. Workshop on Security and Privacy in Spontaneous Interaction and Mobile Phone Use, 2010.

35. Karen P. Tang, Pedram Keyani, James Fogarty, and Jason I. Hong. Putting people in their place: an anonymous and privacy-sensitive approach to collecting sensed data in location-based applications. In Proc. of the SIGCHI conf. on Human Factors in computing systems, 2006.

36. Man Lung Yiu, C.S. Jensen, Xuegang Huang, and Hua Lu. Spacetwist: Managing the trade-offs among location privacy, query performance, and query accuracy in mobile services. In Data Engineering, IEEE 24th Int. Conf. on, 2008.

37. Z. Zhi and Y.K. Choong. Anonymizing geographic ad hoc routing for preserving location privacy. In Distributed Computing Systems Workshops. 25th IEEE Int. Conf. on, 2005.

38. Ge Zhong, Ian Goldberg, and Urs Hengartner. Louis, lester and pierre: three protocols for location privacy. In Proc. of the 7 th int. conf. on Privacy enhancing technologies, 2007. 